\title{
Comparison of the irreversible thermomagnetic behaviour of some ferro- and ferrimagnetic systems
}

\author{
P S ANIL KUMAR ${ }^{\dagger}$, P A JOY* and S K DATE \\ Physical and Materials Chemistry Division, National Chemical Laboratory, Pune 411 008, India \\ ${ }^{\dagger}$ Present Address: Information Storage Technology Group, MESA Research Institute, University of Twente, $7500 \mathrm{AE}$ \\ Enschede, The Netherlands
}

MS received 10 March 1999

\begin{abstract}
The magnetic behaviour of two ferromagnetic oxides and two ferrimagnetic oxides (ferrites) are compared to study the effect of magnetocrystalline anisotropy on thermal-history-dependence of magnetization of these ordered magnetic systems. All four compounds show thermomagnetic irreversibility $\left(M_{\mathrm{FC}}>M_{\mathrm{ZFC}}\right)$ below a certain temperature, $T_{\text {irr. }}$ The highly anisotropic ferromagnetic oxide, $\mathrm{SrRuO}_{3}$ and the hard ferrite, $\mathrm{SrFe}_{12} \mathrm{O}_{19}$ show sharp peaks below $T_{\mathrm{c}}$ in their $M_{\mathrm{ZFC}}(T)$ curves, whereas for the soft ferrite $\mathrm{Ni}_{0.8} \mathrm{Zn}_{0.2} \mathrm{Fe}_{2} \mathrm{O}_{4}$ and the low anisotropic ferromagnetic oxide $\mathrm{La}_{0.7} \mathrm{Ca}_{0.3} \mathrm{MnO}_{3}$ only a broad maximum is observed in $M_{\mathrm{ZFC}}$, when measured in small magnetic fields. The shapes of the $M_{\mathrm{ZFC}}(T)$ curves are inversely-related to the magnitude of the coercivities $\left(H_{\mathrm{c}}\right)$ of the compounds in relation to the applied field, and the temperature dependence of $H_{\mathrm{c}}$. $M_{\mathrm{FC}}$ and $M_{\mathrm{ZFC}}$ are related through the coercivity for all four magnetic systems.
\end{abstract}

Keywords. Ferromagnet; ferrimagnet; thermomagnetic irreversibility; magnetocrystalline anisotropy; coercivity.

\section{Introduction}

Spin glasses are frustrated magnetic systems characterized by their peculiar time and thermal-history-dependent magnetic behaviour. The spins are frozen in random directions due to lack of long-range magnetic interactions, when the system is cooled through its freezing temperature, $T_{\mathrm{f}}$, to a temperature $T<T_{\mathrm{f}}$. Thermal irreversibility between field-cooled (FC) and zero-field-cooled (ZFC) susceptibilities below $T_{\mathrm{f}}$, with a cusp in the low-field $\chi_{\mathrm{ZFC}}$ as well as in the ac susceptibility, long time relaxation of magnetization, etc are the characteristic properties of a spin glass (Mydosh 1993). Nishigori et al (1998) have recently reported that for the ferrimagnetic compounds, TbPdIn and DyPdIn, the field-cooled (FC) and zero-fieldcooled (ZFC) magnetizations show thermal irreversibility below the ferrimagnetic ordering temperature concluding that the irreversibility is due to the appearance of spinglass state. The origin of this spin-glass state is attributed to spin frustrations, due to the rare earth ions forming a deformed Kagome lattice. Similarly, while Maignan et al (1997) have reported the spin-glass-like behaviour of $\mathrm{Pr}_{0.7} \mathrm{Ca}_{0.3} \mathrm{MnO}_{3}$ from low-field susceptibility studies, neutron-diffraction data of the compound evidence a ferromagnetic component. Itoh et al (1994) have concluded that there is no true long-range ferromagnetic order in $\mathrm{Sr}$ -

*Author for correspondence rich compositions in the $\mathrm{La}_{1-x} \mathrm{Sr}_{x} \mathrm{CoO}_{3}$ system, and that they are cluster glasses. Long-time relaxation of $M_{\mathrm{ZFC}}$, large difference between $M_{\mathrm{FC}}$ and $M_{\mathrm{ZFC}}$ below a characteristic temperature, and the absence of magnetic saturation are indicated as evidences for the cluster glass behaviour of compositions with $x>0 \cdot 18$. Similar spin-glass-like properties have been reported recently for other magnetic systems also (Greedan et al 1996; Pecher et al 1999) for which neutron diffraction studies indicate long-range magnetic ordering. The origin of the spin-glass-like nature of these magnetic systems is generally attributed to the competing ferromagnetic and antiferromagnetic exchange interactions, structural disorder, etc.

Our results from a comparison of the low field magnetic behaviours of the ferromagnetic oxides $\mathrm{La}_{0.7} \mathrm{Ca}_{0.3} \mathrm{MnO}_{3}$ and $\operatorname{Pr}_{0.7} \mathrm{Ca}_{0.3} \mathrm{MnO}_{3}$ have indicated that the properties similar to that of a spin glass, observed for $\operatorname{Pr}_{0.7} \mathrm{Ca}_{0.3} \mathrm{MnO}_{3}$, are due to its relatively larger magnetocrystalline anisotropy compared to that of $\mathrm{La}_{0.7} \mathrm{Ca}_{0.3} \mathrm{MnO}_{3}$ (Anil Kumar et al 1998a). The magnetic behaviour of $\mathrm{La}_{0.5} \mathrm{Sr}_{0.5} \mathrm{CoO}_{3}$ similar to that of a cluster glass as reported (Itoh et al 1994), could also be explained based on the high anisotropy of the compound (Anil Kumar et al 1998b). Roy et al (1994, 1997) reported the thermomagnetic irreversibility of different ordered magnetic systems, and concluded that the features of these ordered systems which are similar to those of spin glasses are due to a combination of intrinsic disorder along with inherent anisotropy. In this paper we have compared the low-field FC 
and $\mathrm{ZFC}$ magnetic behaviours of the ferromagnetic oxides, $\mathrm{SrRuO}_{3}$ and $\mathrm{La}_{0.7} \mathrm{Ca}_{0.3} \mathrm{MnO}_{3}$, and the ferrimagnetic oxides, $\mathrm{Ni}_{0.8} \mathrm{Zn}_{0.2} \mathrm{Fe}_{2} \mathrm{O}_{4}$ and $\mathrm{SrFe}_{12} \mathrm{O}_{19}$, having widely differing magnetocrystalline anisotropies. $\mathrm{SrRuO}_{3}$ is a well-known ferromagnetic oxide with very high magnetocrystalline anisotropy (Kabayasi 1976) and there is no magnetic saturation even at very high magnetic fields and low temperatures, whereas $\mathrm{La}_{0.7} \mathrm{Ca}_{0.3} \mathrm{MnO}_{3}$ easily saturates in low magnetic fields and its calculated ferromagnetic moment is comparable to the expected value (Hwang et al 1995). Similarly, the magnetocrystalline anisotropy of the hexagonal ferrite $\mathrm{SrFe}_{12} \mathrm{O}_{19}$ (hard ferrite) is very high (Smit and Wijn 1959; Goldman 1990) compared to that of the cubic ferrite $\mathrm{Ni}_{0 \cdot 8} \mathrm{Zn}_{0 \cdot 2} \mathrm{Fe}_{2} \mathrm{O}_{4}$ (soft ferrite).

\section{Experimental}

The methods of synthesis and characterization of polycrystalline samples of $\mathrm{SrRuO}_{3}(\mathrm{SRH}), \mathrm{La}_{0.7} \mathrm{Ca}_{0.3} \mathrm{MnO}_{3}$ (LCM), $\mathrm{Ni}_{0.8} \mathrm{Zn}_{0.2} \mathrm{Fe}_{2} \mathrm{O}_{4}(\mathrm{NZF})$ and $\mathrm{SrFe}_{12} \mathrm{O}_{19}$ (SRF) have been reported previously (Kulkarni et al 1989; Anil Kumar et al 1996, 1998c; Joy et al 1997). Magnetic measurements (field-cooled (FC) and zero-field-cooled (ZFC) magnetizations and magnetic hysteresis) were performed using a EG\&G PAR model 4500 vibrating sample magnetometer. Magnetic hysteresis measurements (maximum field $=15 \mathrm{kOe}$ ) were performed after cooling the sample through its $T_{\mathrm{c}}$ to the required temperature in zero magnetic field.

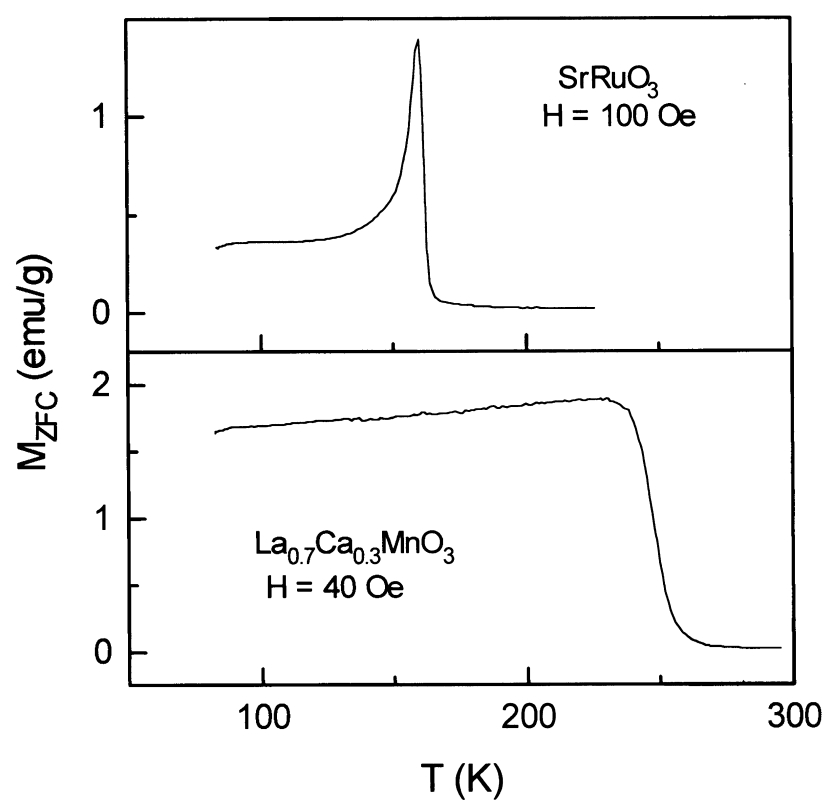

Figure 1. Temperature dependence of $M_{\mathrm{ZFC}}$ of $\mathrm{SrRuO}_{3}$ and $\mathrm{La}_{0.7} \mathrm{Ca}_{0.3} \mathrm{MnO}_{3}$ measured at the low magnetic fields indicated.

\section{Results and discussion}

Temperature variation of the zero-field-cooled magnetizations $\left(M_{\mathrm{ZFC}}\right)$ of $\mathrm{SrRuO}_{3}(\mathrm{SRH}), \mathrm{La}_{0.7} \mathrm{Ca}_{0.3} \mathrm{MnO}_{3}$ (LCM), $\mathrm{Ni}_{0.8} \mathrm{Zn}_{0.2} \mathrm{Fe}_{2} \mathrm{O}_{4}(\mathrm{NZF})$ and $\mathrm{SrFe}_{12} \mathrm{O}_{19}(\mathrm{SRF})$, measured at low magnetic fields, are shown in figures 1 and 2. For LCM and NZF, $M_{\mathrm{ZFC}}$ increases slowly with increasing temperature and shows a broad maximum below the Curie temperature. $M_{\mathrm{ZFC}}$ of SRF decreases slowly as the temperature is increased, a broad minimum is observed between 523 and $623 \mathrm{~K}$ (see inset of figure 2) and again slowly increases up to $\sim 730 \mathrm{~K}$ above which a drastic increase in the magnetization is observed. A sharp peak is obtained at $737 \mathrm{~K}$ followed by the transition to paramagnetic state at $T_{\mathrm{c}}=740 \mathrm{~K}$. Similar sharp peak is observed for SRH also at $160 \mathrm{~K}$ close to its $T_{\mathrm{c}}=161 \mathrm{~K}$.

The magnetic hysteresis behaviour of the ferro- and ferrimagnetic compounds are shown in figure 3 . The coercivities of the low anisotropy compounds, LCM and $\mathrm{NZF}$, are very small compared to the coercivities of SRH and SRF with very large magnetocrystalline anisotropies $\left(H_{\mathrm{c}} \approx 2 K_{1} / M_{\mathrm{s}}\right.$, where $K_{1}$ is the anisotropy constant and $M_{\mathrm{s}}$ is saturation magnetization (Brown 1962)). Temperature dependence of coercivities $\left(H_{\mathrm{c}}\right)$ of the ferromagnetic and ferrimagnetic compounds are compared in figures 4 and 5, respectively. The coercivity of LCM decreases linearly from a value of $35 \mathrm{Oe}$ at $82 \mathrm{~K}\left(T_{\mathrm{c}}=245 \mathrm{~K}\right)$, as the temperature is increased, whereas for SRH, the coercivity is very high $(\sim 3500 \mathrm{Oe})$ at low temperatures. Similarly, for NZF the coercivity decreases from $21 \mathrm{Oe}$ at $323 \mathrm{~K}$ $\left(T_{\mathrm{c}}=750 \mathrm{~K}\right)$ compared to the high coercivity of SRF at

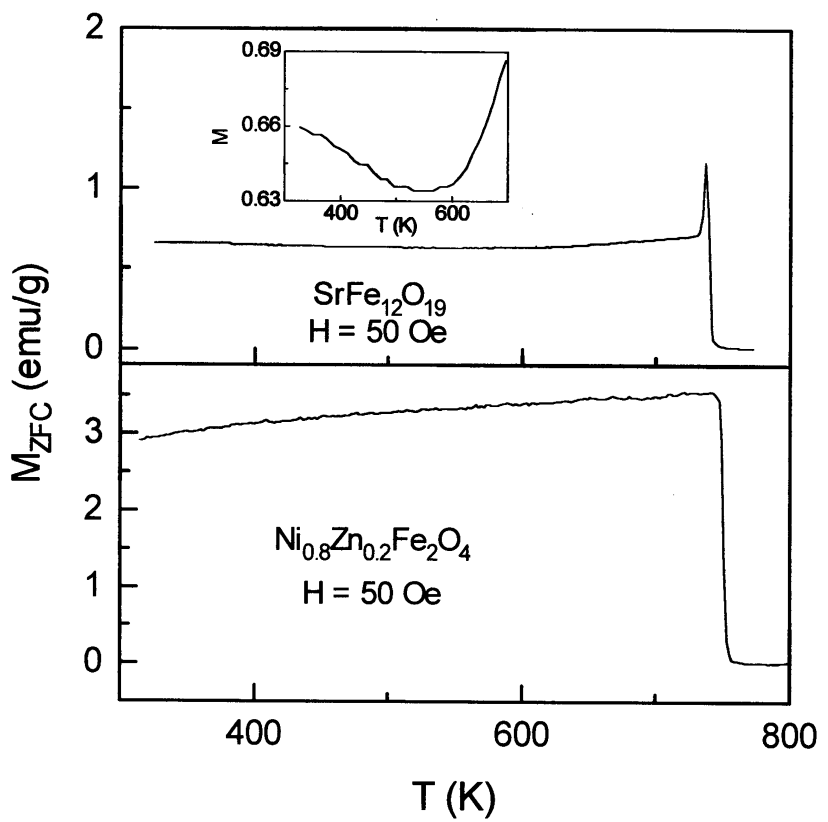

Figure 2. Temperature dependence of $M_{\mathrm{ZFC}}$ of $\mathrm{SrFe}_{12} \mathrm{O}_{19}$ and $\mathrm{Ni}_{0.8} \mathrm{Zn}_{0.2} \mathrm{Fe}_{2} \mathrm{O}_{4}$ measured at the low magnetic fields indicated. Inset: expanded $M_{\mathrm{ZFC}}(T)$ curve of $\mathrm{SrFe}_{12} \mathrm{O}_{19}$. 
room temperature (Jahn and Muller 1969). $H_{\mathrm{c}}$ of SRF increases from $\sim 4000 \mathrm{Oe}$ at $323 \mathrm{~K}$ to $\sim 5500 \mathrm{Oe}$ at $473 \mathrm{~K}$ and decreases further to $\sim 4000 \mathrm{Oe}$ at $673 \mathrm{~K}$, as the temperature is increased.

A comparison of figures 1 and 4 and 2 and 5 shows that for all the four compounds, the temperature dependence of the low-field $M_{\mathrm{ZFC}}$ is inversely related to the magnitude

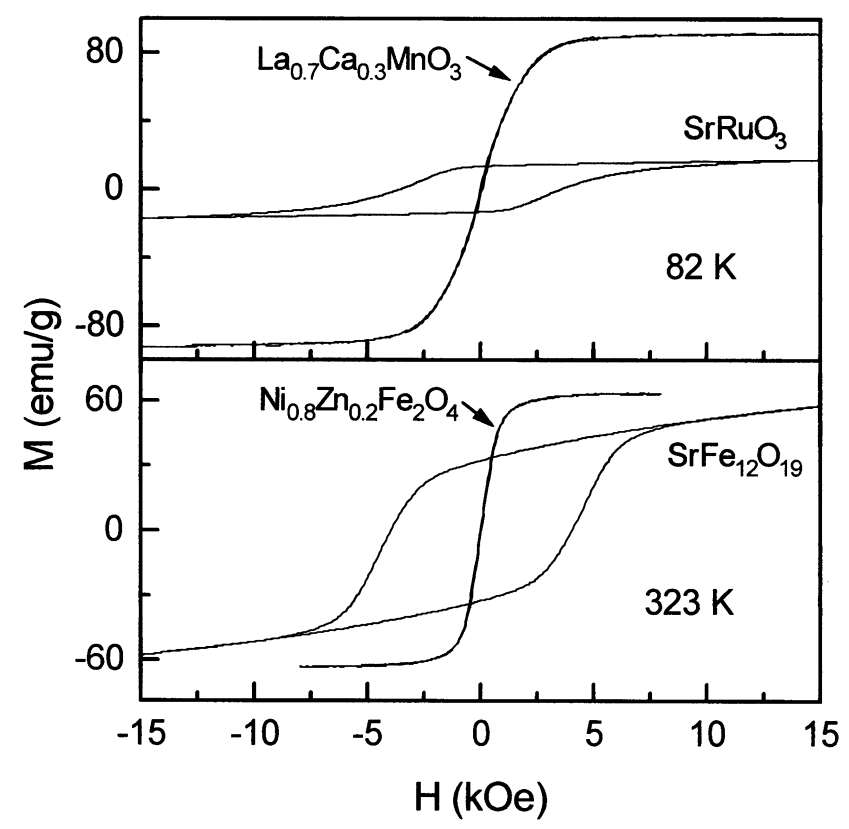

Figure 3. Magnetic hysteresis curves of $\mathrm{SrRuO}_{3}, \mathrm{La}_{0.7} \mathrm{Ca}_{0.3} \mathrm{MnO}_{3}$, $\mathrm{SrFe}_{12} \mathrm{O}_{19}$ and $\mathrm{Ni}_{0.8} \mathrm{Zn}_{0.2} \mathrm{Fe}_{2} \mathrm{O}_{4}$ recorded at the temperatures indicated.

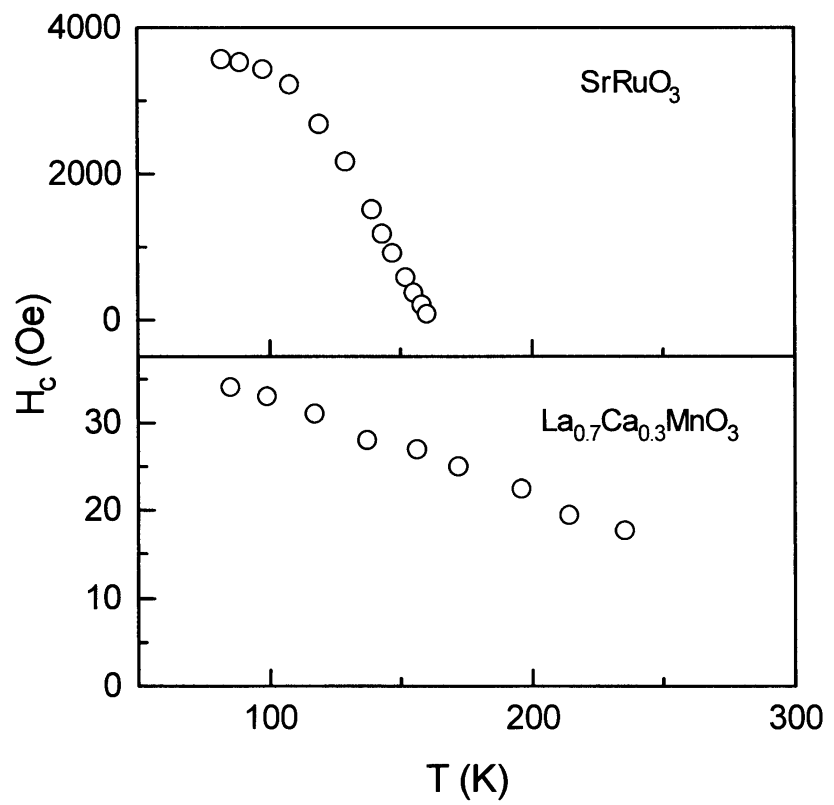

Figure 4. Temperature dependence of $H_{\mathrm{c}}$ of $\mathrm{SrRuO}_{3}$ and $\mathrm{La}_{0.7} \mathrm{Ca}_{0.3} \mathrm{MnO}_{3}$. of the coercivities of the compounds in relation to the applied field, and the temperature dependence of the coercivity. For NZF and LCM the coercivity decreases very slowly with increasing temperature and $M_{\mathrm{ZFC}}$ increases slowly as the temperature is increased. For SRF, $M_{\mathrm{ZFC}}$ decreases up to about $473 \mathrm{~K}$ and again increases up to $730 \mathrm{~K}$ as the temperature is increased. A reverse trend is observed for $H_{\mathrm{c}}$ in this temperature region, showing maximum $H_{\mathrm{c}}$ at $\sim 473 \mathrm{~K}$. This relation between the temperature variations of $H_{\mathrm{c}}$ and $M_{\mathrm{ZFC}}$ of SRF may be clearly seen in figure 2 where the expanded $M_{\mathrm{ZFC}}$ curve measured at $50 \mathrm{Oe}$ is shown as inset. Above $723 \mathrm{~K}$, the coercivity decreases very fast up to the $T_{\mathrm{c}}$ and the $M_{\mathrm{ZFC}}$ curve shows a drastic increase up to $737 \mathrm{~K}$. A sharp peak is observed in $M_{\mathrm{ZFC}}$ at this temperature. For SRH also, a similar trend is observed between $M_{\mathrm{ZFC}}$ and $H_{\mathrm{c}}$. As the applied magnetic field is increased, the peak in the $M_{\mathrm{ZFC}}$ curves becomes broader and the peak temperature is shifted to lower temperatures, as shown for SRF and SRH in figure 6. The difference between $T_{\mathrm{c}}$ and temperature at which a maximum is observed in $M_{\mathrm{ZFC}}(T)$ is related to the magnitude of the applied field and the coercivity at that temperature. The peak in $M_{\mathrm{ZFC}}$ is observed at a temperature where the coercivity is larger than the applied field and a broad maximum is observed when $H_{\mathrm{c}}$ is comparable or smaller than the applied field. This observation shows the influence of the coercivity on the shape of the $M_{\mathrm{ZFC}}$ curves.

FC and ZFC magnetization curves of LCM at $H_{\text {app }}=100 \mathrm{Oe}$ and SRH at $H_{\text {app }}=2000$ Oe are shown in figure 7 and that of NZF at $H_{\text {app }}=50$ Oe and SRF at $H_{\text {app }}=2000$ Oe are shown in figure 8 . All the compounds

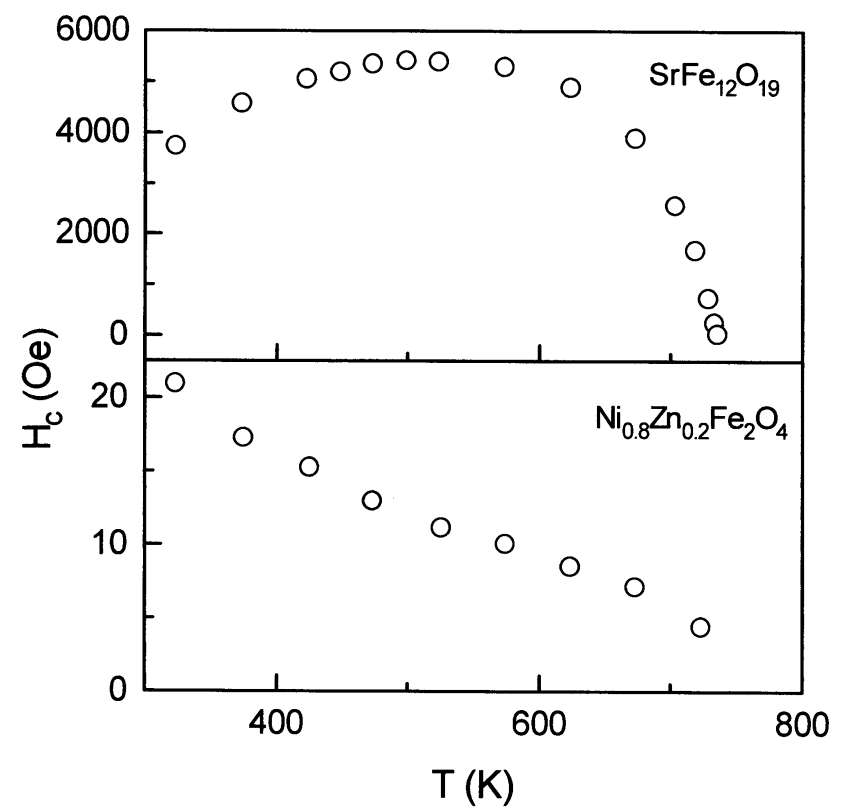

Figure 5. Temperature dependence of $H_{\mathrm{c}}$ of $\mathrm{SrFe}_{12} \mathrm{O}_{19}$ and $\mathrm{Ni}_{0.8} \mathrm{Zn}_{0.2} \mathrm{Fe}_{2} \mathrm{O}_{4}$. 
show thermomagnetic irreversibility $\left(M_{\mathrm{FC}}>M_{\mathrm{ZFC}}\right)$ below a certain temperature, $T_{\text {irr }}$. Furthermore, with increasing applied field, there is a corresponding decrease in the value of $T_{\text {irr }}$, as observed for $\mathrm{La}_{0.5} \mathrm{Sr}_{0.5} \mathrm{CoO}_{3}$ (Anil Kumar et al $1998 \mathrm{~b}) . M_{\mathrm{FC}}$ and $M_{\mathrm{ZFC}}$ of different magnetic systems were found to be related through the expression (Joy et al 1998)

$$
M_{\mathrm{ZFC}} \approx M_{\mathrm{FC}} \frac{H_{\mathrm{app}}}{H_{\mathrm{app}}+H_{\mathrm{c}}},
$$

where $H_{\text {app }}$ is the applied field and $H_{\mathrm{c}}$ the coercivity. $M_{\mathrm{ZFC}}$ values calculated using the above expression from the measured $M_{\mathrm{FC}}$ at different temperatures are shown in figures 7 and 8 . It may be seen that the magnitudes of the calculated $M_{\mathrm{ZFC}}$ at different temperatures are comparable to that of the measured $M_{\mathrm{ZFC}}$ for LCM and NZF. For SRH and SRF also, the shapes of the calculated $M_{\mathrm{ZFC}}$ curves are identical to those of the measured $M_{\mathrm{ZFC}}$, though there are slight differences in their magnitudes. This difference between the calculated and measured $M_{\mathrm{ZFC}} \mathrm{S}$ may be attributed to the presence of a small residual net magnetic field during the process of zero field cooling (Joy et al 1998). A small net magnetic field present during the zero field cooling will greatly affect the $M_{\mathrm{ZFC}}$ of the highly anisotropic compounds than that of the low anisotropy compounds because the magnitude of the measured $M_{\mathrm{ZFC}}$ at low temperatures is determined by the magnetic anisotropy.

The results show that $M_{\mathrm{ZFC}}$ is related to $M_{\mathrm{FC}}$ through the coercivity, and the difference between $M_{\mathrm{FC}}$ and $M_{\mathrm{ZFC}}$ (i.e.

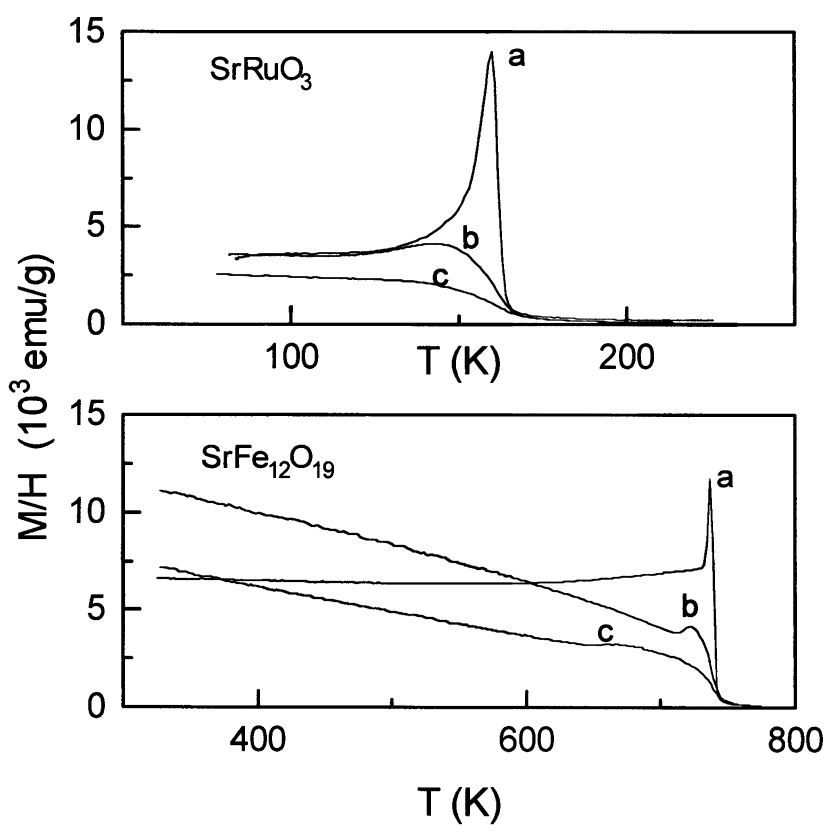

Figure 6. Temperature dependence of $M_{\mathrm{ZFC}} / H$ of $\mathrm{SrFe}_{12} \mathrm{O}_{19}$ and $\mathrm{SrRuO}_{3}$, measured at different applied fields; $50 \mathrm{Oe}(\mathbf{a})$; 2000 Oe (b); 5000 Oe (c). thermomagnetic irreversibility) is due to the magnetocrystalline anisotropy since coercivity is a measure of the anisotropy. Because of the large magnetocrystalline anisotropy of $\mathrm{SrRuO}_{3}$ and $\mathrm{SrFe}_{12} \mathrm{O}_{19}$, their thermomagnetic irreversibility is much larger and sharp peaks are observed in the $M_{\mathrm{ZFC}}$ curves, whereas the low anisotropy

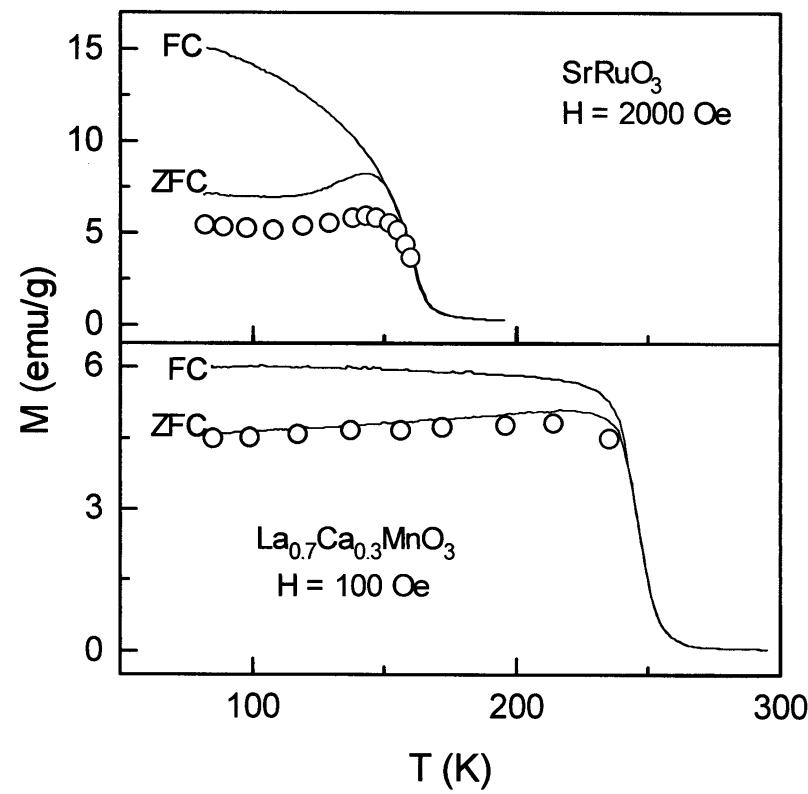

Figure 7. Temperature dependence of $M_{\mathrm{FC}}$ and $M_{\mathrm{ZFC}}$ of $\mathrm{SrRuO}_{3}$ and $\mathrm{La}_{0.7} \mathrm{Ca}_{0.3} \mathrm{MnO}_{3}$ measured at the magnetic fields indicated. The circles represent the calculated $M_{\mathrm{ZFC}}$ from the measured $M_{\mathrm{FC}}$ using the expression given in the text.

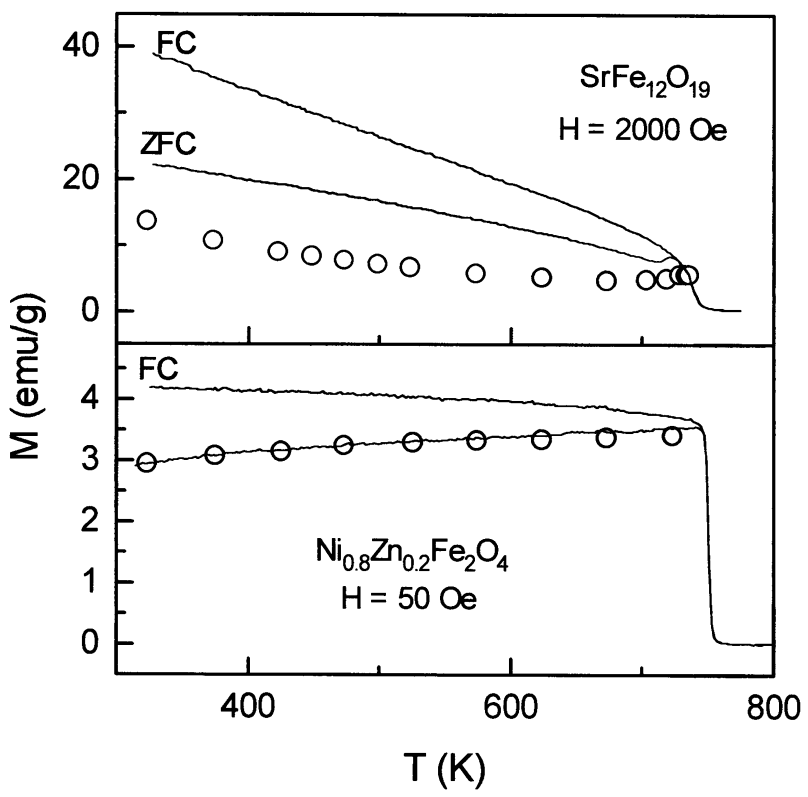

Figure 8. Temperature dependence of $M_{\mathrm{FC}}$ and $M_{\mathrm{ZFC}}$ of $\mathrm{SrFe}_{12} \mathrm{O}_{19}$ and $\mathrm{Ni}_{0.8} \mathrm{Zn}_{0.2} \mathrm{Fe}_{2} \mathrm{O}_{4}$ measured at the magnetic fields indicated. The circles represent the calculated $M_{\mathrm{ZFC}}$ from the measured $M_{\mathrm{FC}}$ using the expression given in the text. 
compounds $\mathrm{La}_{0.7} \mathrm{Ca}_{0.3} \mathrm{MnO}_{3}$ and $\mathrm{Ni}_{0.8} \mathrm{Zn}_{0 \cdot 2} \mathrm{Fe}_{2} \mathrm{O}_{4}$ show broad maximum in their $M_{\mathrm{ZFC}}$ curves and relatively small irreversibility. The shape of the $M_{\mathrm{ZFC}}$ curve is determined by the applied magnetic field strength in relation to the coercive field at a given temperature.

The thermomagnetic irreversibility arises from the magnetic anisotropy due to the difference in the way in which the FC and ZFC measurements are performed on the polycrystalline samples, the sample is either cooled in a magnetic field (FC) or in zero magnetic field (ZFC) through the magnetic ordering temperature. The response of the spins to the external magnetic field depends on the competition between magnetocrystalline anisotropy energy and the applied magnetic field strength. At low measuring fields, compared to the anisotropy field, all the spins will not be oriented in the direction of the applied magnetic field. The magnitude of $M_{\mathrm{ZFC}}$ at low temperatures below the $T_{\mathrm{c}}$ of the compounds will depend on the anisotropy, and therefore the difference between $M_{\mathrm{FC}}$ and $M_{\mathrm{ZFC}}$ will be larger for the highly anisotropic compounds. Hence, the irreversibility between $M_{\mathrm{FC}}$ and $M_{\mathrm{ZFC}}$ and a maximum (cusp or peak) in the ZFC magnetizations of the ferro- and ferrimagnetic systems are due to their magnetic anisotropy and not due to any spin-glass-like behaviour as reported for many ordered magnetic systems.

\section{Conclusions}

The field-cooled and the zero-field-cooled magnetizations of two ferromagnetic compounds, $\mathrm{La}_{0.7} \mathrm{Ca}_{0.3} \mathrm{MnO}_{3}$ and $\mathrm{SrRuO}_{3}$, and two ferrimagnetic compounds, $\mathrm{Ni}_{0.8} \mathrm{Zn}_{0 \cdot 2} \mathrm{Fe}_{2} \mathrm{O}_{4}$ and $\mathrm{SrFe}_{12} \mathrm{O}_{19}$, having widely differing magnetocrystalline anisotropies, are compared. All four compounds show thermal irreversibility between $M_{\mathrm{FC}}$ and $M_{\mathrm{ZFC}} . M_{\mathrm{FC}}$ and $M_{\mathrm{ZFC}}$ are related to each other through the coercivity and applied magnetic field. The extent of irreversibility and the shape of $M_{\mathrm{ZFC}}(T)$ curve at a given applied field are directly related to the coercivity in relation to the magnitude of the applied magnetic field. The magnetic behaviour of $\mathrm{La}_{0.7} \mathrm{Ca}_{0.3} \mathrm{MnO}_{3}$ is similar to that of $\mathrm{Ni}_{0.8} \mathrm{Zn}_{0.2} \mathrm{Fe}_{2} \mathrm{O}_{4}$ which are low anisotropy materials and the properties of the highly anisotropic compounds, $\mathrm{SrFe}_{12} \mathrm{O}_{19}$ and $\mathrm{SrRuO}_{3}$, are comparable to each other.

\section{References}

Anil Kumar P S, Shrotri J J, Kulkarni S D, Deshpande C E and Date S K 1996 Mater. Lett. 27293

Anil Kumar P S, Joy P A and Date S K 1998a Solid State Commun. 10867

Anil Kumar P S, Joy P A and Date S K 1998b J. Phys.: Condens. Matter 10 L487

Anil Kumar P S, Joy P A and Date S K 1998c J. Mater. Chem. 81219

Brown Jr W F 1962 Magnetostatic principles in ferromagnetism (Amsterdam: North-Holland)

Goldman A 1990 Modern ferrite technology (New York: van Nostrand)

Greedan J E, Raju N P, Maignan A, Simon Ch, Pederson J S, Niraimathi A M, Gmelin E and Subramanian M A 1996 Phys. Rev. B54 7189

Hwang H Y, Cheong S-W, Radaelli P G, Marezio M and Batlogg B 1995 Phys. Rev. Lett. 75914

Itoh M, Natori I, Kubota S and Motoya K 1994 J. Phys. Soc. Jpn. 631486

Jahn L and Muller H G 1969 Phys. Status Solidi 35723

Joy P A, Date S K and Anil Kumar P S 1997 Phys. Rev. $B 562324$

Joy P A, Anil Kumar P S and Date S K 1998 J. Phys.: Condens. Matter 1011049

Kabayasi A 1976 J. Phys. Soc. Jpn. 411876

Kulkarni S D, Shrotri J J, Deshpande C E and Date S K 1989 J. Mater. Sci. 243739

Maignan A, Varadaraju U V, Millange F and Raveau B $1997 \mathrm{~J}$. Magn. Magn. Mater. 168237

Mydosh J A 1993 Spin glasses-an experimental introduction (London: Taylor \& Francis)

Nishigori S, Hirooka Y and Ito T 1998 J. Magn. Magn. Mater. 177-181 137

Pecher S, Chevalier B, Laffargne D, Darriet B, Roisnel T and Etourneau J 1999 J. Magn. Magn. Mater. 191282

Roy S B, Pradhan A K and Chaddah P 1994 J. Phys.: Condens. Matter 65155

Roy S B, Pradhan A K, Chaddah P and Sampathkumaran E V 1997 J. Phys.: Condens. Matter 92465

Smit J and Wijn H P J 1959 Ferrites (Eindhoven: Philips Technical Library) 